\title{
GAIT USING MOMENT WITH GRAY AND SILHOUETTE IMAGE
}

\author{
Jyoti Bharti ${ }^{1}$, Navneet Manjhi ${ }^{1}$, M.K.Gupta ${ }^{2}$ and Bimi jain ${ }^{2}$ \\ ${ }^{1}$ Department of Computer Science and Engineering, M.A.N.I.T, Bhopal, India \\ ${ }^{2}$ Department of Electronics and Communication, M.A.N.I.T, Bhopal, India
}

\begin{abstract}
A reliable gait features are required to extract the gait sequences from an images. In this paper suggested a simple method for gait identification which is based on moments. Moment values are extracted on different number of frames of Gray Scale and Silhouette images of CASIA database. These moment values are considered as feature values. Fuzzy logic and Nearest Neighbor Classifier are used for classification. Both achieved higher recognition.
\end{abstract}

\section{KEYWORDS}

Gait, Fuzzy Logic, Nearest Neighbor (without fuzzy), Recognition Rate, Moments.

\section{INTRODUCTION}

A biometric based authentication scheme is a powerful alternative to traditional authentication schemes. A number of biometric characteristics have been used for different application[1],[2],[3],[4],[5],[6]. The term gait was first demonstrate by Johansson in 1970[7]. Gait is a new biometric field to recognize the person at low resolution. While other traits needs higher resolution. It is defined as "A particular way or manner of moving on foot". The human gait generation is a much interesting in fields of biomechanical, robotics, computer animation and visual surveillance etc. The advantage of gait is being difficult to hide, steal or fake[8].

A new gait recognition method is proposed in this paper which is based on moment. Fuzzy inference system and Nearest neighbor classier are used for classification to get better gait recognition rate.

This paper is organized as follows. Section II Summarization of some related existing methods for human gait recognition. Hunan Gait system is described in section III. Section IV provides our proposed method. Experimental results \& analysis are presented in section V, followed by Conclusions \& future scope in Section VI.

\section{Previous Work}

Most of the author worked on different types of images and methods. Liang Wang et. al[9] create silhouette image sequences on subtraction of background image then segmente and track the moving silhouettes of a walking figure. Principal Component Analysis (PCA) was applied to derived time-varying distance signals from a sequence of silhouette images to reduce the dimensionality of the input feature space. Supervised pattern classification techniques are finally

DOI: $10.5121 / \mathrm{ijsc} .2015 .6102$ 
performed in the lower-dimensional eigenspace for recognition. Gait contain shape and dynamic feature which contain useful information for identification. Silhouttes images are used to generated the Gait energy images(GEI), which yield improved performance for identification[10],[11]. A new Procrustes Mean Shape (PMS) was introduced by Yuanyuan Zhang et al.[12]. After created PMS, Compute the similarity between two PMSs. Shape context describes a distribution of all boundary points on a shape with respect to any single boundary point by a histogram of log-polar plot, and offers us a global discriminative characterization of the shape. Standard pattern recognition techniques are used on small and large datasets. It produce effective performance. Different types of classier was used ,most common nearest neighbor (NN) classifier worked on motion contour image (MCI). A time interval between two consecutives local minima of width signal of MCI images produced improved result[13].

\section{Human GaIT}

The definition of Gait is defined as "A peculiar way or manner one walks". Gait recognition is a multistage process[14]. Analysis of walking pattern is a gait cycle. The style of walking or gait cycle of every person is unique[15]. Human gait is the repeated motion of the body parts. Mostly there is no much more changes in head and shoulder motion as compare to hand and legs. The repeated motion part of the body forms a gait cycle. A single Gait cycle or stride is defined as a movement when initial position of a heel comes back again as shown in fig.1. The single gait cycle is further divided into two phases:

1. Stance Phase:Foot in contact with the ground loading response, Mid-stance, Terminal stance, and Pre-swing[16].

2. Swing Phase: Foot not in contact with the ground. Initial swing, Middle swing, and Terminal swing[16].
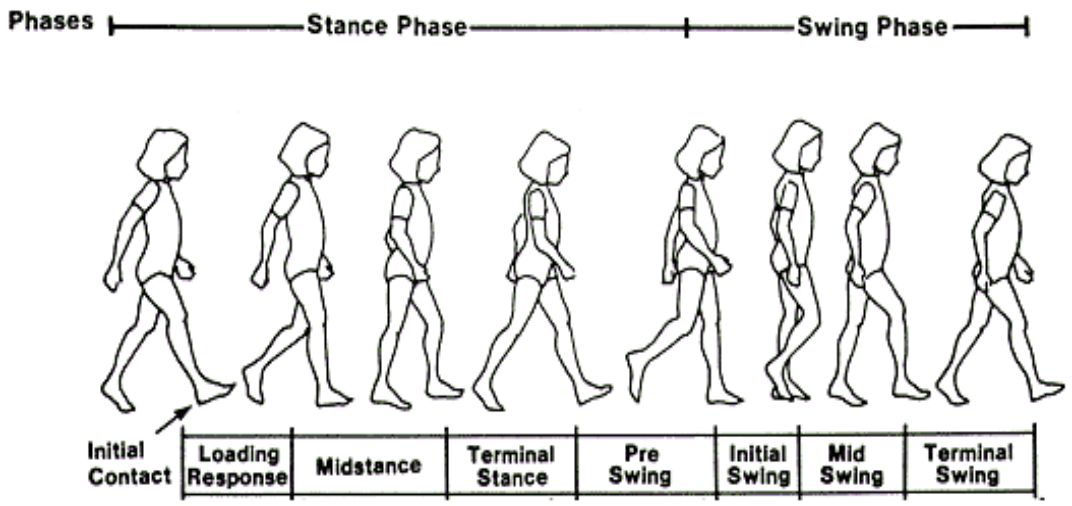

Figure 1. Representation of subject moving through the Gait cycle.

\section{Methodology}

In this paper, a new concept has been used for recognizing the persons on basis of their style of walking. This paper adopted a trouble free method based on Moments which describes the shape and size characteristic of gait. This Moment method is able to find the sequence number of pattern of an object. The methodology is working in two phases:- feature extraction and database creation and classification. 


\subsection{Feature Extraction}

In this phase moment feature values are extracted from Silhouette Images (SI) and Gray Scale Images (GSI). Before feature extraction, the JPEG (Joint Picture Expert Group) color images are changed to gray scale image and Silhouette image is obtained after removing the background from these gray scale images(shown in fig2)[9].

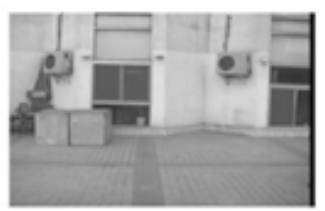

(a)

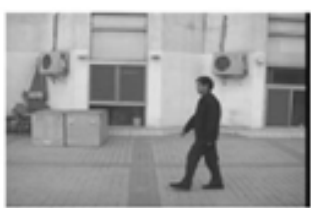

(b)

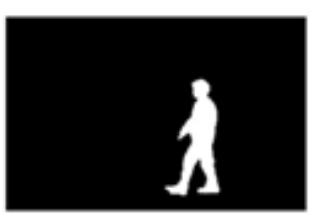

(c)

Figure 2. Examples of moving silhouette extraction and tracking: (a) The background image. (b) An original image,(c) The extracted silhouette.

\subsubsection{Moments}

Moments are area descriptors used to characterize the shape and size of the image. Image $f(x, y)$ is taken as object and grey level of pixel is considered as the mass at a point of the object. For an $N^{*} N$ image, the $(i, j)$ th moment of the image $f(x, y)$ is defined as [17][18].

$$
\mathbf{m}(\mathbf{i}, \mathbf{j})=\sum \mathbf{x} \sum \mathbf{y x i} . \mathbf{y j} \mathbf{f}(\mathbf{x}, \mathbf{y})
$$

The moment feature values are evaluated for gray scale images (GSI) and silhouette images (SI) by using eq. 1 for person image like rj and syj etc as shown in table1.

Table 1. Calculated moment values for GSI \& SI

\begin{tabular}{|l|l|l|}
\hline & Gray scale Result & Silhouette Result \\
\hline User name & I/P Moment & I/P Moment \\
\hline rj & 389282 & 469581 \\
\hline syj & 270945 & 752184 \\
\hline wl & 382716 & 985383 \\
\hline wq & 395101 & 454415 \\
\hline xch & 382491 & 448451 \\
\hline
\end{tabular}

\subsection{Database Creation and Classification}

CASIA Gait data are used for database creation, which is formed by experimentation on 10 users of single side view[19]. The person in image is walking from left to right. Data base is made up of 2 frames. Two different database are generated: first database contains moment values of gray Scale image and second database contains moment values of Silhouette images. Individual databases are created for both types of images.

\subsubsection{Classification}

After extraction of feature values of Silhouette Images and Gray Scale Images two classification methods are used: 
Nearest neighbour Classifier (Without Fuzzy)

Fuzzy Logic Classifier (Mamdani)

\subsubsection{Nearest neighbor Classifier (Without Fuzzy)}

Familiar Euclidean Distance (ED) is used as a nearest neighbour classifier (shown in eq.2). The input moment (mi) values are comparing with the database by using Euclidean Distance. If the ED is smaller than person is identified otherwise not.[20].

Formula for Euclidean Distance between two points (p1, q1).

$$
E D=\sqrt{(q 1-p 1)^{2}}
$$

\subsubsection{Fuzzy logic Classifier}

For Fuzzy classification, a Fuzzy Inference System (FIS) is developed .There are many methods and membership function of Mamdani is most commonly used method for fuzzification and Trapezoidal membership function is used with limit of 0 to 100. Mamdani fuzzification follows the 2-rules and classification is based on these rules. They are connected using with weight-" 1 ", on the basis of these 2-rules and limit can be categorised into the 2-classes-match and no match[21]. The recognition rates for nearest neighbour and fuzzy classifier are categorized in rank-1 and rank-3. Rank-1 is the topmost matching and Rank-3 is the top three matching.

\subsection{Algorithm}

1. Select N-no of frames for input.

2. Color images are converted into gray scale images and Silhouette image.

3. Calculate moment values using formula

$$
\mathbf{m}(\mathbf{i}, \mathbf{j})=\sum \mathbf{x} \sum \mathbf{y x i} . \mathbf{y j} \mathbf{f}(\mathbf{x}, \mathbf{y})
$$

Moment values are the feature values.

4. These feature values are used for recognition. Two Recognition methods are used for each of the above:

a. Without Fuzzy (WF) (Nearest neighbor classifier).

b. Fuzzy Logic (F) (Mamdani).

5. The Fuzzy and without Fuzzy computed are checked and compared with Database. If the mean value is found than the person is garneted to found. If not the person is not found.

6 . Repeat the process for other user.

\section{EXPERIMENTAL RESULTS AND ANALYSIS}

The proposed algorithm is implemented on MATLAB 7.5 using CASIA gait data of 10 users of single side view. For recognition three different numbers of frames are used (1,2 and 3 frames). The database is made using 2 frames for both Gray Scale Image (GSI) and Silhouette image(SI). Then simulation is performed for both images. First Simulation performed by taking randomly any single frame as input, then taking two frames as input and finally three frames are taken as input except database images. Table 2 and 3 shows the simulation results of algorithm for without Fuzzy and Fuzzy respectively using GSI. In this table RR for one, two and three frames for R1 and R3 is shown. The last column of table shows the average RR of all frames for particular rank. 
International Journal on Soft Computing (IJSC) Vol.6, No. 1, February 2015

Table 2. Simulation results of without Fuzzy for GSI

\begin{tabular}{|l|l|l|l|l|l|}
\hline \multicolumn{6}{|l|}{ RR for Gray Scale Image without Fuzzy } \\
\hline \multicolumn{2}{|c|}{} & No. of I/P Frames & \\
\hline \multirow{2}{*}{ RR } & Rank & 1F & 2F & 3F & Avg RR \\
\cline { 2 - 6 } & R3 (\%) & 80 & 100 & 60 & 80 \\
\hline
\end{tabular}

Table 3. Simulation results of Fuzzy for GSI

\begin{tabular}{|l|l|l|l|l|l|}
\hline \multicolumn{5}{|l|}{ RR for Gray Scale Image Fuzzy } & \\
\hline \multicolumn{2}{|c|}{} & \multicolumn{3}{l|}{ No. of I/P Frames } \\
\hline \multirow{2}{*}{ RR } & Rank & $\mathbf{1 F}$ & $\mathbf{2 F}$ & $\mathbf{3 F}$ & Avg RR \\
\cline { 2 - 6 } & R1 (\%) & 80 & 100 & 90 & 90 \\
\cline { 2 - 6 } & R3 (\%) & 100 & 100 & 100 & 100 \\
\hline
\end{tabular}

Fuzzy and Fuzzy for R1 is $60 \%$ and $80 \%$ respectively and for R3 minimum RR is $80 \%$ for without Fuzzy and 100\% for Fuzzy. The Maximum RR for R1 and R3 without Fuzzy and Fuzzy is $100 \%$.

From tables 4 and 5 showing the simulation results of algorithm for without Fuzzy and Fuzzy respectively As per the tables 2 and 3, it is observed that the minimum RR for GSI without using Silhouette image. In this table RR for one, two and three frames of R1 and R3 is shown. The last column of tables shows the average RR of all frames for particular rank.

Table 4. Simulation results of without Fuzzy for Silhouette Image.

\begin{tabular}{|l|l|l|l|l|l|}
\hline \multicolumn{6}{|l|}{ RR for Silhouette Image without Fuzzy } \\
\hline \multirow{2}{*}{} & No. of I/P Frames & \\
\hline \multirow{2}{*}{ RR } & Rank & $\mathbf{1 F}$ & $\mathbf{2 F}$ & $\mathbf{3 F}$ & Avg RR \\
\cline { 2 - 6 } & R3 (\%) & 60 & 80 & 80 & 73.33333 \\
\hline
\end{tabular}

Table 5. Simulation results of Fuzzy for Silhouette Image.

\begin{tabular}{|l|l|l|l|l|l|}
\hline \multicolumn{6}{|l|}{ RR for Silhouette Image with Fuzzy } \\
\hline \multirow{2}{*}{ Rank } & No. of I/P Frames & \\
\hline \multirow{2}{*}{ RR } & R1 (\%) & 1F & 2F & 3F & Avg RR \\
\cline { 2 - 6 } & R3 (\%) & 90 & 80 & 100 & 90 \\
\hline
\end{tabular}

From tables 4 and 5, it is observed that the minimum RR for Silhouette image without Fuzzy and Fuzzy for R1 is $60 \%$ and $80 \%$ respectively and for R3 minimum RR is $80 \%$ for without Fuzzy and $100 \%$ for Fuzzy. The Maximum RR for R3 without Fuzzy and Fuzzy is 100\%, and for R1 without Fuzzy is $80 \%$ and $100 \%$ for Fuzzy.

Following figures 2 and 3 shows the graphical representation of without Fuzzy RR for R1 of GSI and Silhouette image. 
International Journal on Soft Computing (IJSC) Vol.6, No. 1, February 2015

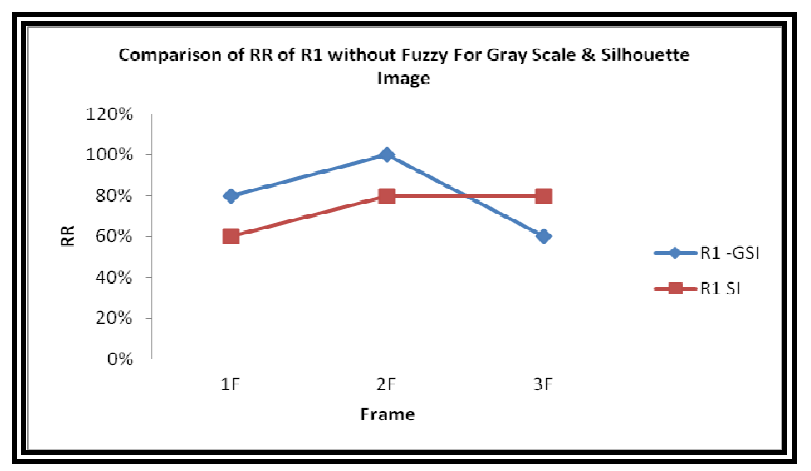

Figure 2. Comparison of RR of R1 without Fuzzy For Gray Scale \& Silhouette Image

As per figure 2 it is observed that GSI and Silhouette both generates higher RR for 2-frames and GSI is better than SI for 1 and 2-frames but for 3-frames SI is better

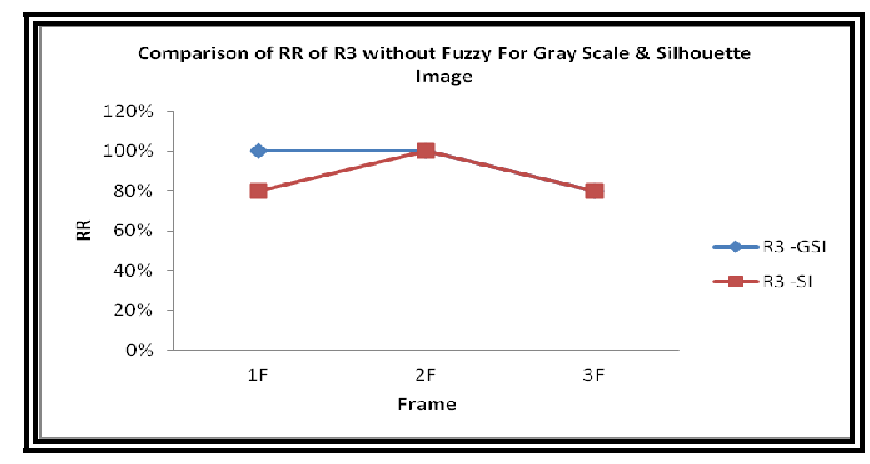

Figure 3. Comparison of RR of R3 without Fuzzy For Gray Scale \& Silhouette Image.

From analysis of figure 3, it is found that GSI and Silhouette both generates higher RR for 2frames and GSI is better than SI for 1 and but for 2 and 3-frames both produce same RR.

Following figures 4 and 5 shows the pictorial representation of Fuzzy RR for R3 of GSI and Silhouette image

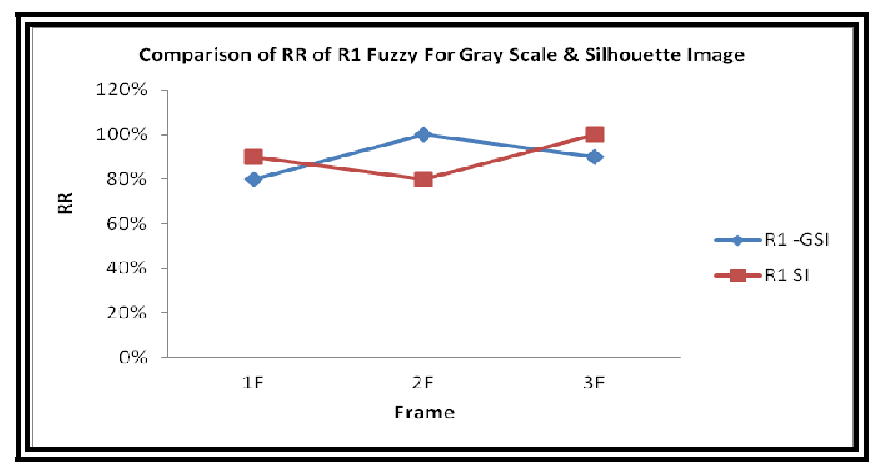

Figure 4. Comparison of RR of R1 Fuzzy For Gray Scale \& Silhouette Image. 
As per figure 4 it is observed that for GSI and Silhouette overall performance is almost same. GSI generates $100 \% \mathrm{RR}$ at 2-frames but SI generates 100\% RR at 3-frames. Overall 3-frames are better for Fuzzy.

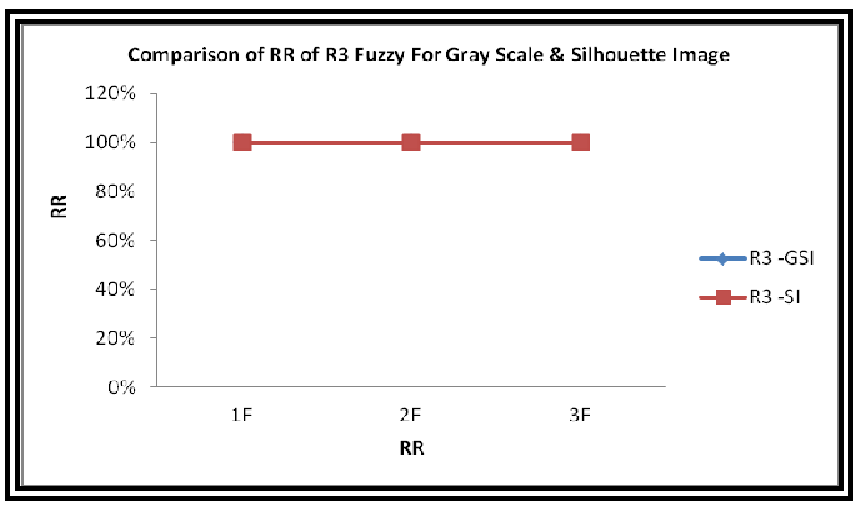

Figure 5. Comparison of RR of R3 Fuzzy For Gray Scale \& Silhouette Image.

From figure 5 it is observed that GSI and Silhouette both generates $100 \%$ RR for all frames.

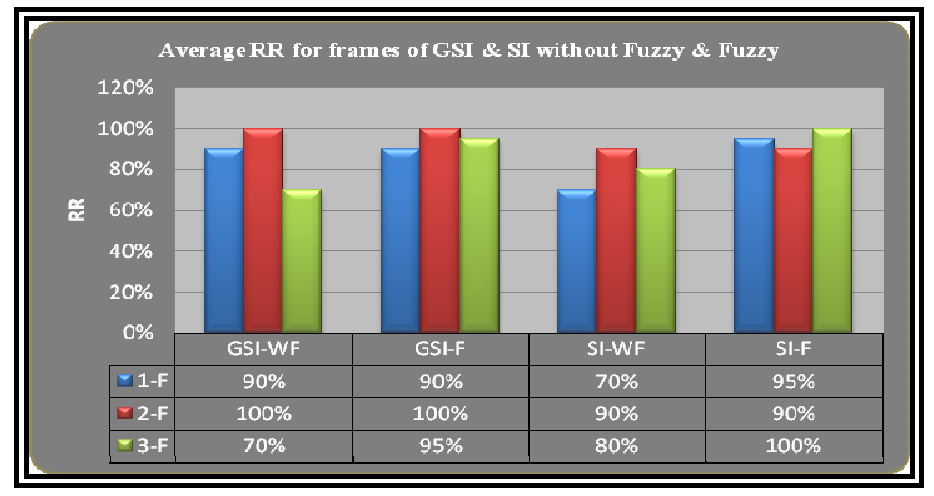

Figure 6. Average RR For GSI \& SI without Fuzzy \& Fuzzy.

Figure 6 is graphical comparison of performance of GSI and SI using fuzzy without fuzzy. 2frames RR is better than that of 1 and 3. 3-frames RR is higher than 1-frames RR for Both GSI and SI without Fuzzy and Fuzzy method for all frames.

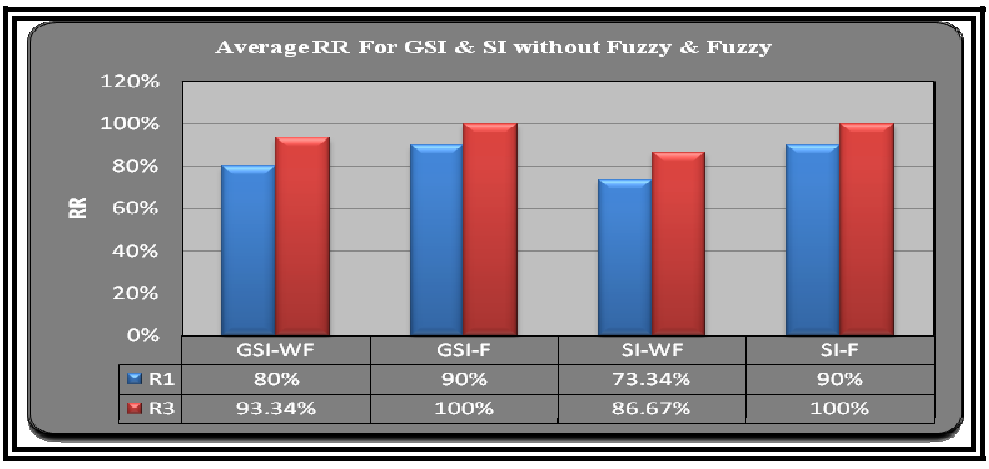

Figure 7. Average RR For GSI \& SI without Fuzzy \& Fuzzy. 
Figure 7 is a graphical comparison of performance of without Fuzzy and Fuzzy method for for GSI and SI images. This figure illustrate that Fuzzy is better than without Fuzzy and R3 generates higher RR than R1. RR for Fuzzy of GSI and SI is same but for without Fuzzy GSI is better than SI.

Table 6. Best frame and RR for Fuzzy and without Fuzzy (R1 \& R3)

\begin{tabular}{|l|l|l|l|l|}
\hline Image Type & \multirow{2}{*}{ Classifier } & \multicolumn{2}{|l|}{ Avg RR } & Best \\
\cline { 3 - 4 } & & R1 (\%) & R3 (\%) & \\
\hline \multirow{2}{*}{ FSI } & WF & 80 & 93.34 & $2-F$ \\
\cline { 2 - 5 } & F & 90 & 100 & $2-F$ \\
\hline \multirow{2}{*}{ SI } & WF & 73.34 & 86.67 & $2-F$ \\
\cline { 2 - 5 } & F & 90 & 100 & $3-F$ \\
\hline
\end{tabular}

As per-observations of fig. 2-7 and Table 2-6, its find that Fuzzy is better than without Fuzzy and RR of R3 is higher as compare to R1. For R1 and R3 RR of Fuzyy is higher than without Fuzzy for all frames. RR for GSI is higher than SI. Overall 2-frames generates higher RR.

\section{COMPARATIVE ANALYSIS}

Table 7 shows the comparison of implemented method with other methods. From this table it is clear that highest RR for Rank-1 is achieved by proposed method (90\%) with Fuzzy logic classifier and for Rank-3 highest RR is (100\%). By without Fuzzy classifier RR for Rank-1 is also better (80\%) and for Rank-3 (93.34\% RR) is achieved. For Rank-5 100\% RR achieved by without Fuzzy and Fuzzy. The table shows the implemented method gives higher RR a compare to other previous methods

Table 7. Comparison of R1, R3 \& R5 RR of our method with others.

\begin{tabular}{|c|c|c|c|c|c|}
\hline \multicolumn{3}{|l|}{ Method } & $\begin{array}{l}\text { Rank- } \\
1(\%)\end{array}$ & $\begin{array}{l}\text { Rank-3 } \\
(\%)\end{array}$ & $\begin{array}{l}\text { Rank-5 } \\
(\%)\end{array}$ \\
\hline \multicolumn{3}{|c|}{ Dynamic VarianceFeatures [22] } & $76 \%$ & - & \\
\hline \multicolumn{3}{|c|}{$\begin{array}{l}\text { Silhouette Analysis-Based Gait } \\
\text { Recognition for Human Identification } \\
\text { [9] }\end{array}$} & $75 / 82.5$ & - & $97 / 100$ \\
\hline \multicolumn{3}{|c|}{$\begin{array}{l}\text { Human Gait Recognition using All } \\
\text { Pair Shortest Path[23] }\end{array}$} & 75 & & 95 \\
\hline \multirow{4}{*}{$\begin{array}{l}\text { Our } \\
\text { method }\end{array}$} & \multirow[t]{2}{*}{ GSI } & WF & 80 & 93.34 & 100 \\
\hline & & $\mathrm{F}$ & 90 & 100 & 100 \\
\hline & \multirow[t]{2}{*}{ SI } & WF & 73.34 & 86.67 & 100 \\
\hline & & $\mathrm{F}$ & 90 & 100 & 100 \\
\hline
\end{tabular}

\section{CONCLUSION AND FUTURE WORK}

It is concluded from the presented methodology, Moment feature values of Gray Scale and Silhouette image also achieved better recognition rate for fuzzy and without fuzzy. Without Fuzzy of GSI has $80 \%$ RR and $93.3 \%$ RR is better to SI of R1 and R3 but for fuzzy GSI and SI both have same( $90 \%$ \& 100\%)RR for R1 and R3.

R3 RR for both Fuzzy and without Fuzzy is higher to R1.

Two-frames produce better results as compare to 1 and 3-frames. 
Fuzzy is better than without Fuzzy for R1 and R3.

Future Work: In present work only one side is consider. Extract the feature values for other side views and other types of costumes like long gown and saree etc. Combine the gait with the other biometric traits like finger, face, palm etc recognition can be used for network security.

\section{REFERENCES}

[1] Jain, Anil K., Ross, Arun., Pankanti, Sharath (2006) "Biometrics: A Tool for Information Security", IEEE Transactions on Information Forensics and Security, Vol. 1, No. 2, pp21-38.

[2] Jain, Anil, K., Ross, Arun., Prabhakar, Salil (2004) “An introduction to biometric recognition", IEEE Transactions on circuits and systems for video technology, Vol. 14, No. 1, pp67-80.

[3] Liu, Ling-Feng., Jial, Wei., Zhu, Yi-Hai (2009) "Survey of Gait Recognition”, Springer ICIC 2009, LNAI 5755, pp652-659.

[4] Pushparani, M., Sasikala, D (2012) "A Survey of Gait Recognition Approaches Using PCA \& ICA", Global Journal of Computer Science and Technology, Vol. 12 Issue 10 Version 1.0.

[5] Soriano, Maricor., Araullo, Alessandra., Saloma, Caesar (2004) "Curve spreads-a biometric from front-view gait video", Elsevier journal of Pattern Recognition Letters, Vol. 25, pp1595-1602.

[6] Su, Han., Huang, Feng-Gang (2005) "Human Gait Recognition Based On Motion Analysis", IEEE Proceedings of the Fourth International Conference on Machine Learning and Cybernetics, Guangzhou, Vol. 7, pp4464-4468.

[7] Johnsson, G (1973) "Visual perception of biological motion and a model for its Analysis, Perception Psychophys", IEEE conference on intelligent control and automa, Vol. 14(2) 201-211.

[8] Boulgouris, Nikolaos V., Hatzinakos, Dimitrios., Plataniotis, Konstantinos, N (2005) "Gait Recognition: A challening signal processing technology for biometric identification”, IEEE Signal Processing Magazine, pp56-70 November.

[9] Wang, Liang., Tan, Tieniu., Ning, Huazhong., Hu, Weiming (2003) "Silhouette Analysis-Based Gait Recognition for Human Identification", IEEE Transaction on Pattern Analysis and Machine intelligent, Vol. 25, No. 12, pp1505- 1518.

[10] Bashir, Khalid., Xiang, Tao., Gong, Shaogang (2008) "Feature selection on gait energy image for human identification", IEEE International Conference on Acoustics, Speech, and Signal Processing, pp44-50.

[11] Han, Ju., Bhanu, Bir (2006) "Individual Recognition Using Gait Energy Image", IEEE Transaction on Pattern Analysis and Machine Intelligence, Vol. 28, No. 2, pp316-322.

[12] Zhang, Yuanyuan., Yang, Niqing., Li, Wei., Wu, Xiaojuan., Ruan, Qiuqi (2010) "Gait Recognition Using Procrustes Shape Analysis and Shape Context”, Springer Accv2009, Part III, LNCS 5996, pp256-265.

[13] Lee, Byungyun., Hong, Sungjun., Lee, Heesung., Kim, Euntai (2010) "Gait Recognition System using Decision-Level Fusion”, 5th IEEE conference on industrial electronics and application, pp.313-316.

[14] Wang, Junqiu., Makihara Yasushi., Yagi, Yasushi (2008) "Human Tracking and Segmentation Supported by Silhouette-based Gait Recognition”, IEEE International Conference Robotics and Automation(ICRA), pp1698 - 1703.

[15] Lu, Haiping., Plataniotis, K.N., Venetsanopoulos, A.N (2006) "Layered Deformable Model for Gait Analysis", 7th IEEE International Conference on Automatic Face and Gesture Recognition, pp. 249256.

[16] Boulgouris, Nikolaos V., Chi, Zhiwei X (2007) "Human gait recognition based on matching of body components”, Elsevier journal of pattern recognition, Vol. 40, pp1763 - 1770.

[17] Jain, Bimi., Gupta, M.K., Bharti, Jyoti (2012) "Efficient Iris Recognition algorithm using Method of Moments", International Journal of Artificial Intelligence \& Applications (IJAIA), Vol. 3, No.5.

[18] Dhananjay, K., Theckedath (2005) Image Processing, Using Matlab Codes, Nandu Publishers.

[19] Center for Biometrics and Security Research (CBSR).

[20] Gonzalez, Rafael, C., Woods, Richard, E (2009) Digital Image Processing, Pearson Education.

[21] Lamba, V.K (2008) Neuro Fuzzy Systems, Laxmi publication.

[22] Ren, Yanmei Chai Jinchang., Zhao Rongchun., Jia, Jingping (2006) "Automatic Gait Recognition using Dynamic Variance Features", IEEE conference on automatic face and gesture recognition, pp475-480

[23] Bharti, Jyoti., Gupta, M. K (2011) "Human Gait Recognition using All Pair Shortest Path", International Conference on Computing and Computer Vision(ICCCV 2011), Vol. 9, pp279-284 